\title{
Role of Diffusion Tensor Imaging as an Independent Predictor of Cognitive and Language Development in Extremely Low-Birth-Weight Infants
}

\author{
U. Pogribna, K. Burson, R.E. Lasky, P.A. Narayana, P.W. Evans, and N.A. Parikh
}

\begin{abstract}
BACKGROUND AND PURPOSE: Diffusion tensor imaging at term can predict later development of cerebral palsy. Less is known about its ability to independently predict cognitive and language development in extremely preterm infants. The goals of the study were to investigate the following: 1) whether regional DTI measures at term-equivalent age in extremely low-birth-weight infants (birth weight, $\leq 1000 \mathrm{~g}$ ) are predictive of Bayley III developmental scores at 18- to 22-months' corrected age, and 2) to compare white matter microstructural development at term and neurodevelopmental outcomes of extremely low-birth-weight infants with healthy term controls.
\end{abstract}

MATERIALS AND METHODS: Fractional anisotropy and mean diffusivity in 7 vulnerable cerebral regions were measured in 42 extremely low-birth-weight and 16 term infants with high-quality DTI scans. The Bayley mental scale score (average of cognitive and language scale scores) was the primary outcome of interest with individual scores serving as secondary outcomes. Multiple linear regression modeling was used to identify the incremental ability of DTI measures to predict Bayley scores over known predictors.

RESULTS: Compared with healthy term infants, extremely low-birth-weight infants exhibited significantly higher mean diffusivity and lower fractional anisotropy in 6 of 7 regions. At 18- to 22-months' corrected age, 39 extremely low-birth-weight infants (93\%) and 14 term infants (88\%) had undergone neurodevelopmental assessments. Although not statistically significant, extremely low-birth-weight infants averaged 7-9 points lower on Bayley subtests than term controls. In multivariable analyses, centrum semiovale mean diffusivity was a significant predictor of mental and language scale scores, and subventricular zone fractional anisotropy was a significant predictor of cognitive scale scores. A $10 \%$ increase in centrum semiovale mean diffusivity was associated with a $4.6(95 \% \mathrm{Cl}, 1.6-7.6)$ point lower mental scale score (adjusted $R^{2}=0.341, P=.001$ ).

CONCLUSIONS: In our extremely low-birth-weight cohort, DTI was an independent predictor of later cognitive and language development.

ABBREVIATIONS: ELBW = extremely low-birth-weight ( $\leq 1000 \mathrm{~g}$ birth weight); $\mathrm{FA}=$ fractional anisotropy; $\mathrm{GA}=$ gestational age; $\mathrm{MD}=$ mean diffusivity

$\mathbf{U}^{\mathrm{p}}$ p to $50 \%$ of extremely preterm infants are diagnosed with cognitive impairment by school age. ${ }^{1}$ While cerebral white matter abnormalities visible on cranial sonography account for an

Received May 30, 2013; accepted after revision July 24

From the Division of Neonatology (U.P., K.B., R.E.L., P.W.E., N.A.P.), Department of Pediatrics, and Department of Radiology (P.A.N.), University of Texas Health Science Center, Houston, Texas; Center for Perinatal Research (N.A.P.), The Research Institute at Nationwide Children's Hospital, Columbus, Ohio; and Department of

Pediatrics (N.A.P.), Ohio State University College of Medicine, Columbus, Ohio.

Author contributions: conceived and designed the experiments: N.A.P., R.E.L.,

P.A.N.; performed the experiments: N.A.P., K.B., P.W.E., U.P.; analyzed the data: U.P., N.A.P.; wrote the paper: U.P., N.A.P.; critically reviewed the paper: U.P., K.B., R.E.L., P.A.N., P.W.E., N.A.P.

This work was supported by the National Center for Research Resources grant UL RR024148 (University of Texas Health Science Center at Houston Center for Clinical and Translational Sciences) and the National Center for Research Resources/ Eunice Shriver Kennedy National Institute of Child Health \& Human Development, grant UL1 RR024148-04S3 (Best Pharmaceuticals for Children Act). The 3T scanner was partially funded by National Center for Research Resources/National Institutes of Health through a grant to P.A.N. (grant S10 RR19186). The funding agencies important proportion of those with later impairment, nearly $30 \%$ with no abnormality on sonography develop impairment. ${ }^{2}$ The increasingly common finding of diffuse noncystic white matter signal abnormalities, primarily visible only by using conventional brain MR imaging, has been linked with later cognitive impairment. ${ }^{3-5}$ However, neurodevelopmental outcome prediction by using qualitative conventional MR imaging at term-equivalent age remains suboptimal. ${ }^{5-7}$ More accurate risk prediction at term

played no role in the design, conduct, or analysis of the trial. The authors take full responsibility for the integrity of the data and analyses.

Paper previously presented at: Neonatology General Poster Session at the Pediatric Academic Societies Annual Meeting, May 4-7, 2013; Washington, DC (Abstract, Publication 3832.535).

Please address correspondence to Nehal A. Parikh, D.O., M.S., Center for Perinatal Research, Research Building III, 575 Children's Crossroad, Columbus, $\mathrm{OH} 43215$; e-mail: Nehal.Parikh@NationwideChildrens.org

- Indicates open access to non-subscribers at www.ajnr.org

http://dx.doi.org/10.3174/ajnr.A3725 
could facilitate targeted intensive early intervention therapies and novel neuroprotection trials.

Recent MR imaging advances permit quantification of water diffusion and anisotropy in vivo by using DTI. This advancement has facilitated sensitive detection of microstructural white matter injury and aberrant brain development in extremely preterm infants, making DTI a powerful diagnostic tool and potential early imaging biomarker for cognitive and other neurodevelopmental impairments. ${ }^{8-11}$ There is emerging evidence that DTI can predict neurodevelopmental impairments in very low-birth-weight ( $\leq 1500 \mathrm{~g}$ ) infants. ${ }^{12-15}$ However, studies in more immature extremely low-birth-weight ( $\leq 1000 \mathrm{~g})$ (ELBW) populations are lacking, and the incremental benefits of DTI over conventional MR imaging remain poorly defined. The goals of our study were 2-fold: 1) to investigate whether regional abnormalities on DTI at term-equivalent age in ELBW infants are independent predictors, over known clinical predictors, of standardized cognitive and language scores at 18- to 22-months' corrected age; and 2) to compare white matter microstructural development at term and neurodevelopmental outcomes of ELBW infants with healthy term controls at 18- to 22-months' corrected age by using the newer Bayley Scales of Infant and Toddler Development III. ${ }^{16}$

\section{MATERIALS AND METHODS \\ Participants}

Fifty ELBW and 16 healthy term infants from the Children's Memorial Hermann Hospital neonatal intensive care unit and neonate nursery, respectively, were enrolled to undergo brain DTI at 38 weeks' postmenstrual age or before discharge, if discharge was earlier. Dates of enrollment for ELBW and term infants were May 2007 to July 2009 and July 2008 to January 2010, respectively. The main eligibility criteria for ELBW infants were a birth weight $\leq 1000 \mathrm{~g}$ and survival to 34 weeks postmenstrual age or greater and, for term infants, gestational age (GA) of $\geq 37$ weeks and birth weight appropriate for GA. ELBW infants with known congenital CNS anomalies or who were mechanically ventilated with unstable clinical status at the time of enrollment were excluded. Term infants with any history of perinatal distress or complications or $\geq 42$ weeks' GA were excluded. Institutional review board approval and informed consent were obtained before enrollment.

\section{Image Acquisition and Processing}

We used a 3T Achieva scanner (Philips Healthcare, Best, the Netherlands), equipped with a 32-channel receiver and a gradient system capable of producing gradient amplitudes of $80 \mathrm{mT} / \mathrm{m}$ with a slew rate of $200 \mathrm{~T} / \mathrm{m} / \mathrm{s}$ for all MR imaging. An 8-channel phased array head coil was used for data acquisition. The DTI protocol consisted of a single-shot, spin-echo planar sequence with TR/TE, $6000 / 61 \mathrm{~ms}$; in-plane resolution, $1.6 \times 1.6 \mathrm{~mm}^{2}$; FOV, $180 \mathrm{~mm}^{2}$; $128 \times 128$ matrix; and $2-m m$ contiguous sections. Fifteen directions of diffusion gradients were used with a b-value of 800 $\mathrm{s} / \mathrm{mm}^{2}$; low b-value $=0$; sensitivity encoding factor $=2$. The imaging parameters for the proton-attenuation/T2-weighted scan were the following: TE1, $9 \mathrm{~ms}$; TE2, $175 \mathrm{~ms}$; TR, 10,000 ms; flip angle, $90^{\circ}$; FOV , $180 \times 180 \mathrm{~mm}^{2}$; $256 \times 256 \mathrm{~mm}^{2}$ matrix; section thickness, $2 \mathrm{~mm}$. Sagittal T1, axial FLAIR, axial MPRAGE, coronal T2, and axial gradient-recalled echo sequences were also obtained. Total acquisition time was 4 minutes for the DTI sequence and 25 additional minutes for the conventional sequences. Total imaging time, including repeated sequences, took approximately 1 hour on average.

Patients were fed and swaddled before MR imaging scans. MedVac infant vacuum splint (CFI Medical Solutions, Fenton, Michigan), Insta-Puffy Silicone Earplugs (E.A.R. Inc, Boulder, Colorado), and Natus Mini Muffs (Natus Medical, San Carlos, California) were used for restraint and noise reduction. No sedation was given. All scans were supervised by an experienced neonatologist and a neonatal research nurse.

Image postprocessing and tensor calculations were performed by using MRIStudio software, Version 3.0.3 (Johns Hopkins University, Baltimore, Maryland; http://cmrm.med.jhmi.edu). ${ }^{17} \mathrm{Re}-$ gions of interest were selected on the basis of prior published data. ${ }^{18,19}$ The following white matter ROIs were selected for further analysis: anterior and posterior limbs of the internal capsule, frontal and occipital periventricular zones, the centrum semiovale, the genu and splenium of the corpus callosum, and the subventricular zone. To minimize variability in region-of-interest placement, we used ImageJ, Version 1.44p (National Institutes of Health; http://imagej.nih.gov/ij) for development and placement of region-of-interest templates ( 1 for each region of interest) in the native space (Fig 1). All analyses were blinded to clinical variables and cranial sonography and anatomic MR imaging findings. Fractional anisotropy (FA) and mean diffusivity (MD) were measured for each region of interest.

\section{Follow-Up and Developmental Assessment}

Standardized follow-up assessments of ELBW and term infants were completed at 18- to 22-months' corrected age in the HighRisk Infant Follow-up Clinic at the University of Texas Health Science Center at Houston. During the follow-up visit, all patients had a complete neurologic physical examination to assess gross motor function and ascertain the presence of cerebral palsy by standardized Eunice Kennedy Shriver National Institute of Child Health and Human Development (NICHD) Neonatal Research Network examiners. The Bayley Scales of Infant and Toddler Development III $^{16}$ cognitive and language subtests (scale of 50 to 150 ; mean of 100; 150 indicating the most advanced development) were administered by a masked, certified examiner.

\section{Data Analysis}

Detailed data that included demographics, maternal, perinatal, and neonatal history were prospectively collected for all enrolled ELBW infants during their hospitalization. Additional data in regard to demographics and socioeconomic and health statuses were also collected for infants during their follow-up period. All data were entered into a secure data base with error checks by qualified neonatal research nurses. All unique identifiers were removed to protect privacy and to blind investigators to clinical history.

STATA/IC 12 (StataCorp, College Station, Texas) was used for all data analyses. Two-sample $t$ tests, $\chi^{2}$ tests, and Fisher exact tests were used to describe and compare demographic characteristics of ELBW and term infants, as appropriate. Intrarater reliability 

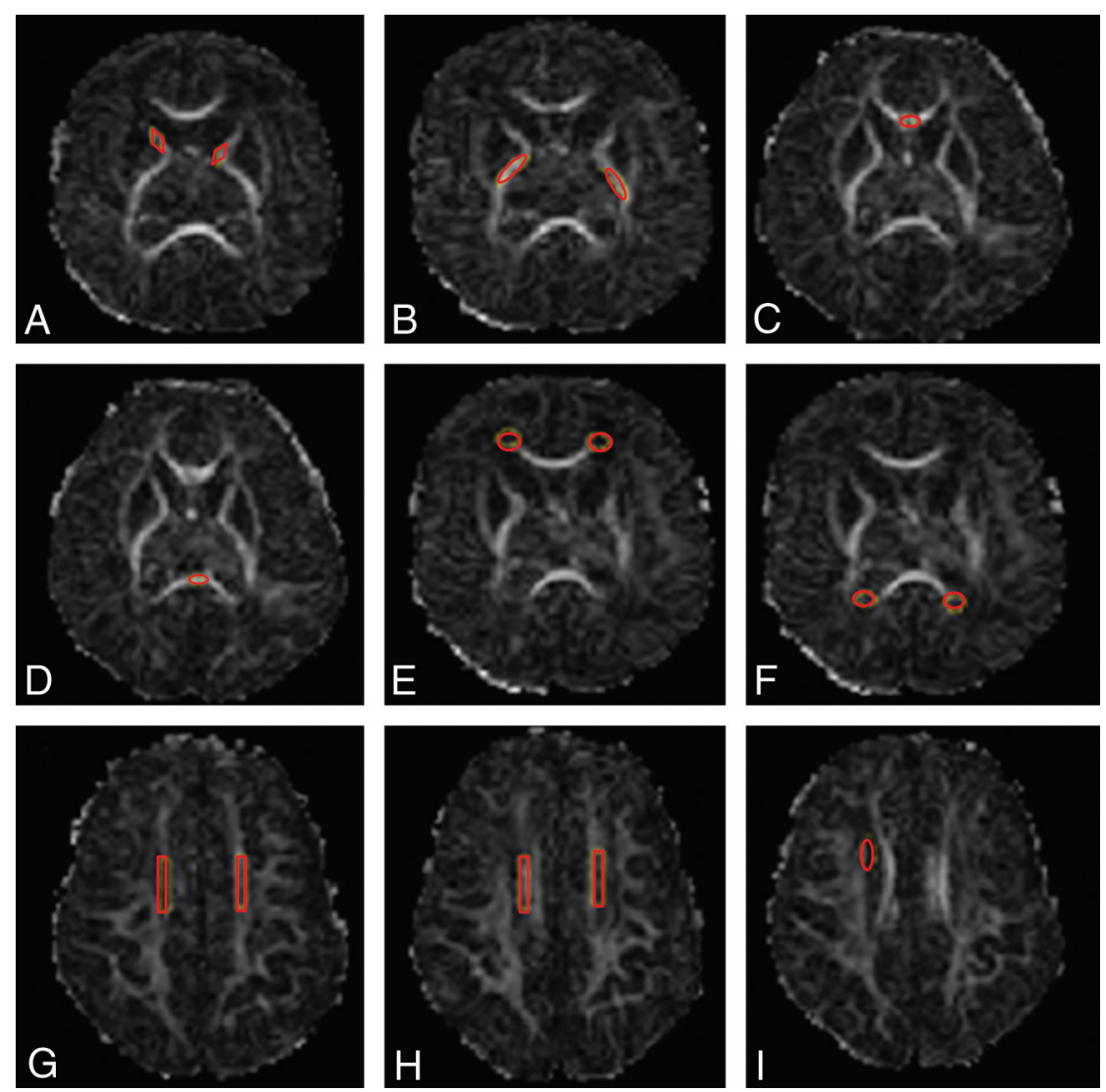

FIG 1. Region-of-interest templates and placements shown on FA maps. A, Anterior limb of the internal capsule, $B$, Posterior limb of the internal capsule. $C$ and $D$, Corpus callosum, genu and splenium. $E$, Frontal periventricular zone. $F$, Occipital periventricular zone. $G$ and $H$, Centrum semiovale at 2 consecutive levels. I, Subventricular zone (right-sided). The same templates were used for all scans.

for region-of-interest placements was assessed by using intraclass correlation coefficients on 17 randomly selected ELBW cases.

The mental scale score (the average of cognitive and language scale scores) on Bayley III was the primary outcome of interest. This average score is an overall composite of cognitive and language abilities and permits comparison with the Bayley II mental scale score. ${ }^{20}$ Bayley III cognitive and language scale scores were also evaluated independently as secondary outcomes. Bayley III scores (mental, cognitive, and language) and FA and MD measures for each region of interest were compared between term and ELBW infants by using a 2-sample $t$ test or a Wilcoxon MannWhitney test, as appropriate.

Postmenstrual age was significantly associated with several regional diffusion measures as well as neurodevelopmental outcomes. As such, all regression analyses were controlled for this important confounder. Only regions in which FA and MD were significantly different in ELBW infants compared with term controls were selected for correlation with Bayley III outcomes. Univariate analyses for each region of interest were correlated with mental, cognitive, and language scale scores in separate analyses. Multiple regression modeling was used to identify the incremental and independent ability of DTI measures to predict Bayley III scores. All significant FA and MD ROIs $(P<.05)$ were further analyzed in adjusted, multiple regression models. Each multiple regression model was adjusted for birth weight, white matter in- jury on cranial sonography (presence of ventriculomegaly with or without blood in the ventricles, echodense lesions in the parenchyma, cystic periventricular leukomalacia, and/or a porencephalic cyst evident on cranial ultrasound before 28 days of life), and abnormal conventional MR imaging findings at term (the presence of signal abnormalities, brain atrophy, and/or abnormal gray matter or white matter maturation for age). All assumptions of linear regression were met. In the final models, $P<.05$ was considered significant. All model results are presented as adjusted $R^{2}$ values, which represent the proportion of variability in the outcome explained by the model and regression coefficients, with corresponding 95\% confidence intervals. Bootstrapping was used to assess the internal validity of the final adjusted models.

\section{RESULTS}

Forty-eight of 50 (96\%) ELBW infants and 14 of $16(88 \%)$ term infants returned for follow-up at 18- to 22-months' corrected age. Forty-two of these 48 ELBW infants and all 14 of the term infants had high-quality DTI scans free of motion artifacts. Complete Bayley testing was unavailable in 3 ELBW (2 had only cognitive scores) and 2 term infants due to behavioral problems, resulting in the final sample size of 39 ELBW and 12 term infants with complete follow-up and high-quality DTI scans. Demographic and clinical characteristics of these infants are presented in Table 1. ELBW infants were significantly smaller for GA (range, 23-30 weeks) and weight (range, 468-1000 g) than their term counterparts; however, postmenstrual age at MR imaging was similar between groups. Both groups were similar in regard to race and insurance status at birth and at follow-up. The 6 ELBW infants excluded secondary to motion artifacts were similar in a majority of key demographic and clinical variables and Bayley scores compared with those with complete follow-up data.

Cerebral palsy was diagnosed in 4 of 39 (10\%) ELBW infants but in none of the term infants at 18- to 22-months' corrected age. Cerebral palsy was not considered as an outcome measure due to our limited study power (Table 1).

Bayley III subtest scores are presented in Table 2. The mean age at follow-up was $20.8 \pm 3.8$ and $20.8 \pm 1.9$ months' corrected age for preterm and term infants, respectively. The mean cognitive score for term controls was close to the test norm of 100 points, but the mean language score was approximately 5 points lower. Although not statistically significant, ELBW infants averaged 7-9 points lower than healthy term controls on the Bayley subtests.

Compared with healthy term infants, ELBW infants exhibited 
Table 1: Demographic and clinical characteristics of study infants

\begin{tabular}{|c|c|c|}
\hline & $\begin{array}{c}\text { Term }(n=12) \\
\text { (Mean) (SD) or (\%) }\end{array}$ & $\begin{array}{c}\text { ELBW }(n=39) \\
\text { (Mean) (SD) or (\%) }\end{array}$ \\
\hline Maternal age (yr) & $23.6(5.6)$ & $27.9(4.9)^{\mathrm{a}}$ \\
\hline GA at birth (wk) & $38.6(1.1)$ & $25.6(1.5)^{\mathrm{a}}$ \\
\hline Birth weight (g) & $3159.8(428.0)$ & $768.6(147.6)^{\mathrm{a}}$ \\
\hline PMA at MRI (wk) & $38.9(1.1)$ & $38.4(2.3)$ \\
\hline Male & $33 \%$ & $54 \%$ \\
\hline Race: white & $42 \%$ & $46 \%$ \\
\hline Private or mix of private/public medical insurance & $33 \%$ & $44 \%$ \\
\hline Maternal education: college degree or greater & $50 \%$ & $72 \%$ \\
\hline $\begin{array}{l}\text { Antenatal steroids given (full course of } \\
\text { betamethasone) }\end{array}$ & $\mathrm{N} / \mathrm{A}$ & $62 \%$ \\
\hline Small for GA (<10th percentile) & $0 \%$ & $18 \%^{\mathrm{a}}$ \\
\hline \multicolumn{3}{|l|}{ Delivery room resuscitation } \\
\hline Intubation at birth & $\mathrm{N} / \mathrm{A}$ & $92 \%$ \\
\hline Resuscitation drug given & $\mathrm{N} / \mathrm{A}$ & $3 \%$ \\
\hline Bronchopulmonary dysplasia & N/A & $85 \%$ \\
\hline Late-onset sepsis ( \pm culture) & N/A & $67 \%$ \\
\hline Culture proven late-onset sepsis & $\mathrm{N} / \mathrm{A}$ & $28 \%$ \\
\hline Necrotizing enterocolitis & N/A & $3 \%$ \\
\hline Major surgery (with general anesthesia) & N/A & $26 \%$ \\
\hline Severe retinopathy of prematurity & $\mathrm{N} / \mathrm{A}$ & $21 \%$ \\
\hline White matter injury on cranial ultrasound ${ }^{\mathrm{b}}$ & N/A & $21 \%$ \\
\hline Abnormal conventional MRI findings at term ${ }^{c}$ & $\mathrm{~N} / \mathrm{A}$ & $66 \%$ \\
\hline \multicolumn{3}{|l|}{ Child's insurance status at time of follow-up } \\
\hline Private & $33 \%$ & $37 \%$ \\
\hline Public medical insurance & $67 \%$ & $63 \%$ \\
\hline Household income $\geq \$ 50,000$ at follow-up & $33 \%$ & $32 \%$ \\
\hline
\end{tabular}

Note:-PMA indicates postmenstrual age; N/A, not applicable.

a $P<.05$.

${ }^{b}$ Defined as any presence of ventriculomegaly with or without blood in the ventricles, echodense lesions in the parenchyma, cystic periventricular leukomalacia, and/or a porencephalic cyst evident on cranial ultrasound prior to 28 days of life.

'Defined as the presence of signal abnormalities, brain atrophy, and/or abnormal gray matter or white matter maturation for age.

Table 2: Developmental outcome comparisons of term and ELBW infants

\begin{tabular}{|c|c|c|c|}
\hline Developmental Outcome & $\begin{array}{c}\text { Term }(n=12) \\
(\text { Mean) }(S D) \text { or }(\%)\end{array}$ & $\begin{array}{c}\text { ELBW }(n=39) \\
(\text { Mean) }(S D) \text { or }(\%)\end{array}$ & $P$ Value \\
\hline Cerebral palsy & $0 \%$ & $10 \%$ & $\mathrm{~N} / \mathrm{A}$ \\
\hline Bayley III mental scale score & $96.4(15.4)$ & $88.5(16.6)$ & .16 \\
\hline Bayley III cognitive scale score & $99.2(17.8)$ & $92.6(15.3)$ & .46 \\
\hline Bayley III language scale score & 94.9 (16.4) & $86.2(18.6)$ & .21 \\
\hline
\end{tabular}

Note:-N/A indicates not applicable.

significantly higher MD and lower FA in 6 of the 7 selected brain regions (Fig 2). Mean FA and MD for term infants were $0.143 \pm$ 0.037 and $1.232 \times 10^{-3} \pm 0.099 \times 10^{-3}$, respectively, in the subventricular zone and $0.157 \pm 0.031$ and $1.530 \times 10^{-3} \pm$ $0.118 \times 10^{-3}$, respectively, in the centrum semiovale. For ELBW infants, mean $\mathrm{FA}$ and $\mathrm{MD}$ in the subventricular zone were $0.118 \pm 0.031$ and $1.577 \times 10^{-3} \pm 0.348 \times 10^{-3}$; and in the centrum semiovale, they were $0.126 \pm 0.028$ and $1.812 \times 10^{-3} \pm$ $0.153 \times 10^{-3}$, respectively. The intrarater intraclass correlation coefficient for region-of-interest placements was 0.93 . In univariate analyses, subventricular zone FA had a significant positive association with mental and cognitive scale scores; the centrum semiovale and subventricular zone MD had a negative association with mental, cognitive, and language scale scores (Table 3). As such, lower FA or higher MD values in these 2 regions were associated with lower Bayley III scores. White matter injury on cranial sonography performed within the first 28 days of birth was also a significant predictor of all 3 Bayley III scores. Abnormal conven- tional MR imaging findings (abnormalities in maturation or myelination, atrophy, and structural lesions) at term were a significant predictor of adverse Bayley mental and language scale scores but not cognitive scale scores at 18- to 22-months' corrected age. No associations between birth weight and Bayley III scores were found in the ELBW cohort.

Centrum semiovale MD and subventricular zone FA remained significant independent predictors of Bayley III scores in the adjusted multiple-regression models. Centrum semiovale MD was the only significant predictor of mental scale scores, our primary outcome (Table 4). In secondary analyses, centrum semiovale MD was predictive of language scale scores, and lower subventricular zone FA values were predictive of adverse cognitive scale scores on the Bayley scale (Table $4)$. In clinical terms, a $10 \%$ increase in centrum semiovale MD was associated with a -4.6 (95\% CI, -7.6 to -1.6$)$ point decrease on mental scale scores and a -4.6 ( $95 \% \mathrm{CI},-8.0$ to -1.2 ) point decrease on language scale scores. Similarly, a $10 \%$ increase in subventricular zone FA correlated with a 24.5 (95\% CI, 9.6-39.3) point increase on the Bayley III cognitive scale score. Most interesting, of the adjusted factors, only white matter injury on cranial sonography remained a significant predictor of lower mental and cognitive scale scores in multivariable regression modeling. Bootstrapping confirmed the internal validity of the 3 adjusted prediction models.

\section{DISCUSSION}

We identified 2 independent regional biomarkers of cognitive and language development in a prospective cohort of ELBW infants imaged at term-equivalent age. Our findings are consistent with emerging evidence of the utility of DTI microstructural abnormalities at term for prediction of neurodevelopmental impairment in very low-birth-weight preterm infants. ${ }^{9,12-14}$ Our data support the value of DTI in ELBW infants and suggest that FA and MD are independent predictive biomarkers of early cognitive and language development, in addition to cranial sonography and conventional MR imaging measures.

Cerebral palsy and results of standardized neurodevelopmental assessments are now the most commonly reported outcome measures in studies of preterm infants. Our small sample size, however, precluded us from testing cerebral palsy as an end point. Cognitive and language assessments may be considered the most clinically meaningful outcomes, with up to $50 \%$ of ELBW infants developing cognitive impairments by 2 years of age. ${ }^{1}$ Mental scale scores and language and cognitive scale scores on the Bayley III were our primary and secondary outcomes of interest, respec- 
tively, serving as markers of functional brain development in our cohort of ELBW infants. Several studies have found similar associations between DTI and cognitive and language development, though these studies were completed in older preterm infants during childhood and adolescence. ${ }^{21-23}$

Our study is one of a few that has attempted to correlate early DTI measurements at term with later cognitive development. ${ }^{21-23}$ The subventricular zone and centrum semiovale were 2 vulnerable regions of particular interest-abnormalities in fractional anisotropy (lower values) and mean diffusivity (higher values) in these regions were associated with lower mental, cognitive, and
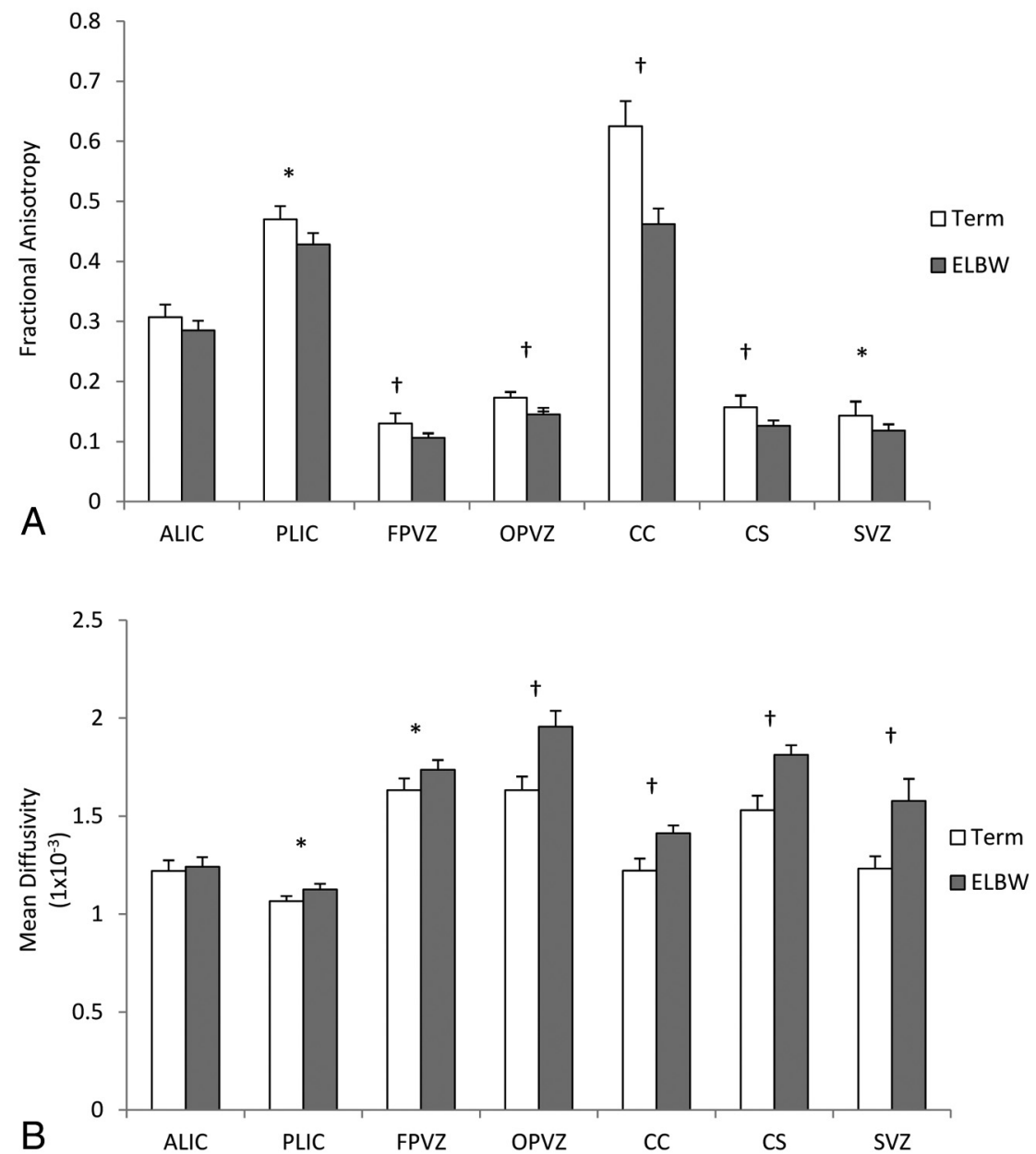

FIG 2. Comparison of mean (SD) fractional anisotropy $(A)$ and mean diffusivity (SD) $(B)$ in term (white) and ELBW (gray) infants in selected regions of interest. Asterisk indicates $P<.05$; dagger, $P<.01$; ALIC, anterior limb of the internal capsule; PLIC, posterior limb of the internal capsule; FPVZ, frontal periventricular zone; OPVZ, occipital periventricular zone; CC, corpus callosum; $\mathrm{CS}$, centrum semiovale; SVZ, subventricular zone. language scale scores. These abnormalities remained significant predictors of Bayley III scores, even after adjustment for known adverse predictors of outcome (Table 3). Because diffuse excessive high signal abnormalities are especially common in the centrum semiovale, our results provide additional evidence that such diffuse white matter abnormalities are predictive of cognitive impairment. ${ }^{4,5,13}$ Even relatively small changes (10\%)_equivalent to $1.1-3.5$ weeks of brain maturation ${ }^{24}$ - in fractional anisotropy or mean diffusivity, respectively, were associated with clinically meaningful effects on Bayley scores at 18- to 22- months' corrected age. Our results highlight the importance of measuring both $\mathrm{MD}$ and FA in vulnerable regions, and differences we observed in correlations with outcome suggest differences in underlying white matter pathology. Mean diffusivity measures the average water diffusion, and high values are believed to reflect destruction of tissue architecture. In contrast, FA values reflect the degree of water anisotropy along different axes; decreased tissue anisotropy likely reflects tissue degeneration. ${ }^{25}$

In addition to numerous animal models of CNS diseases, ${ }^{26,27}$ there are now human fetal $^{28}$ and adult studies ${ }^{29,30}$ that have demonstrated accurate correlations between DTI microstructural measures and histopathology measures. Increasing fractional anisotropy and decreasing mean diffusivity are markers of improved axonal organization and white matter myelination and are associated with increasing brain development. ${ }^{31}$ Multiple studies to date, however, have shown that preterm infants exhibit both macro- and microstructural differences in their white matter organization, often irrespective of underlying injury, compared with healthy term infants; such differences persist into early childhood, adolescence, and even adulthood. ${ }^{18,23,24,32}$ In their study, Shim et $\mathrm{al}^{33}$ observed that chronic lung disease and postnatal infection in preterm infants were independently correlated with altered FA in the posterior limb of the internal capsule and corpus callosum, suggesting that factors other than prematurity

Table 3: Univariate analyses of DTI and clinical variables and their association with Bayley III scores at 18- to 22-months' corrected age in ELBW infants

\begin{tabular}{|c|c|c|c|c|c|c|}
\hline \multirow[b]{2}{*}{ Variable } & \multicolumn{2}{|l|}{ Mental Score } & \multicolumn{2}{|l|}{ Cognitive Score } & \multicolumn{2}{|l|}{ Language Score } \\
\hline & Coefficient $(95 \% \mathrm{Cl})$ & $P$ & Coefficient $(95 \% \mathrm{Cl})$ & $P$ & Coefficient $(95 \% \mathrm{Cl})$ & $P$ \\
\hline FA subventricular zone & $179.4(13.8-345.0)$ & .04 & $219.4(62.8-376.1)$ & .01 & $141.1(-57.1-339.2)$ & .16 \\
\hline MD subventricular zone & $-18.9(-33.4$ to -4.3$)$ & .01 & $-17.5(-32.0$ to -3.1$)$ & .02 & $-20.2(-37.1$ to -3.3$)$ & .02 \\
\hline MD centrum semiovale & $-55.8(-86.7$ to -24.9$)$ & .001 & $-51.4(-82.3$ to -20.4$)$ & .002 & $-60.2(-96.3$ to -24.1$)$ & .002 \\
\hline Birth weight & $0.01(-0.02-0.1)$ & .42 & $0.01(-0.03-0.04)$ & .80 & $0.01(-0.03-.04)$ & .80 \\
\hline White matter injury on cranial US & $-16.8(-29.1$ to -4.5$)$ & .01 & $-15.7(-27.9$ to -3.5$)$ & .01 & $-15.7(-27.9$ to -3.5$)$ & .01 \\
\hline Abnormal findings on conventional MRI & $-11.6(-22.3$ to -0.9$)$ & .03 & $-5.8(-16.8-5.1)$ & .29 & $-5.8(-16.8$ to 5.1$)$ & .29 \\
\hline
\end{tabular}

Note:-US indicates ultrasound. 
Table 4: Abnormalities in MD and FA and their association with Bayley III scores at 18-22 months corrected age in ELBW infants Clinical/DTI Variable Coefficient $(95 \% \mathrm{CI}) \quad P$

\begin{tabular}{ccc}
\hline A) Mental scale score & & \\
(adjusted $R^{2}$ 0.34/model P 0.001) & & \\
MD centrum semiovale & $-45.7(-75.6$ to -15.7$) .004$ \\
Birth weight & $0.01(-0.02-0.04)$ & .41 \\
White matter injury on ultrasound & $-12.5(-23.9$ to -1.0$)$ & .03 \\
Abnormal MRI findings at term & $-5.0(-14.9-4.8)$ & .31 \\
B) Language scale score & & \\
$\quad$ (adjusted $R^{2}$ 0.37/model P 0.001) & & \\
MD centrum semiovale & $-46.4(-80.1$ to -12.8$)$ & .01 \\
Birth weight & $0.02(-0.02-0.054)$ & .28 \\
White matter injury on ultrasound & $-12.0(-24.9-1.0)$ & .07 \\
Abnormal MRI at term & $-10.5(-21.7-0.7)$ & .07 \\
C) Cognitive scale score & & \\
(adjusted $R^{2}$ 0.30/model P 0.003) & & .002 \\
FA subventricular zone & $244.6(96.1-393.1)$ & .07 \\
Birth weight & $0.01(-0.02-0.05)$ & .40 \\
White matter injury on ultrasound & $-14.0(-25.5$ to -2.6$)$ & .02 \\
Abnormal findings on MRI at term & $-5.5(-15.6-4.6)$ & .28 \\
\hline
\end{tabular}

alone may affect brain microstructure in preterm infants. Similarly, we have found an independent association between increasing duration of mechanical ventilation (our proxy for chronic lung disease) and delayed maturation (increased MD) of the occipital periventricular zone and centrum semiovale. ${ }^{34}$

Studies in more mature preterm infants found FA and MD to be sensitive markers of microstructural white matter abnormalities and significantly associated with neurodevelopmental impairments. ${ }^{9,13,14}$ For example, Krishnan et al $^{13}$ observed increasing centrum semiovale MD to be associated with a decreasing developmental quotient in infants $\leq 34$ weeks' GA at birth, similar to our primary outcome findings. However, van Kooij et $\mathrm{al}^{14}$ reported a significant association between corpus callosum FA and Bayley III cognitive scores in infants $<31$ weeks' GA, a finding we were not able to validate. Study differences in DTI acquisition and processing methodology (eg, region of interest-based versus whole-brain analyses) and cohort characteristics may well explain such a difference in results. Our data further highlight the importance of DTI measures as early imaging biomarkers and useful adjuncts to conventional neuroimaging to predict cognitive and language development, particularly in the smallest and most vulnerable infants.

The main characteristics of our study included a high-risk population, consistent and reproducible DTI measurements by using robust postprocessing methods, high-resolution 3T imaging, internal validation by using bootstrap, and high follow-up rates. However, our relatively small sample size may have resulted in type II errors (false-negatives). In particular, the lack of a statistical difference in neurodevelopmental scores we observed between ELBW and term infants may have been largely affected by our limited study power. Our decision to avoid sedation for MR imaging in any of the study infants likely contributed to the $10 \%$ rate of excessive motion artifacts and case exclusions. Additionally, 18- to 22-months' corrected age is a relatively early follow-up interval for cognitive assessments because these appear to be more accurate at school age. ${ }^{35}$ Despite these limitations, our DTI biomarkers accounted for a sizable percentage of the variance in cognitive and language scores, in addition to qualitative neuroimag- ing approaches, and warrant validation in a larger cohort with school-age follow-up.

\section{CONCLUSIONS}

In this prospective cohort study of ELBW infants, DTI microstructural biomarkers at term-equivalent age were independent predictors, in addition to known clinical and imaging risk factors, of cognitive and language development at 18- to 22-months' corrected age. Larger cohort studies with longer follow-up are needed to further assess the predictive validity of DTI measures as early imaging biomarkers of functional impairments in extremely preterm infants.

\section{ACKNOWLEDGMENTS}

We sincerely thank Vipulkumar S. Patel for assistance with MR imaging data acquisition as well as the families and nurses that made this study possible.

Disclosures: Ponnada A. Narayana—RELATED: Grant: National Institutes of Health,* UNRELATED: Grants/Grants Pending: National Institutes of Health.* Nehal A. Parikh—RELATED: Grant: Eunice Kennedy Shriver National Institute of Child Health and Human Development, ${ }^{*}$ National Center for Research Resources, ${ }^{*}$ Comments: grants UL1 RR024148 and UL1 RR024148-04S3. *Money paid to the institution.

\section{REFERENCES}

1. Potharst ES, van Wassenaer AG, Houtzager BA, et al. High incidence of multi-domain disabilities in very preterm children at five years of age. J Pediatr 2011;159:79-85

2. Laptook AR, O'Shea TM, Shankaran S, et al, for the NICHD Neonatal Network. Adverse neurodevelopmental outcomes among extremely low birth weight infants with a normal head ultrasound: prevalence and antecedents. Pediatrics 2005;115:673-80

3. Volpe JJ. Cerebral white matter injury of the premature infant: more common than you think. Pediatrics 2003;112:176-80

4. Dyet LE, Kennea N, Counsell SJ, et al. Natural history of brain lesions in extremely preterm infants studied with serial magnetic resonance imaging from birth and neurodevelopmental assessment. Pediatrics 2006;118:536-48

5. Iwata S, Nakamura T, Hizume E, et al. Qualitative brain MRI at term and cognitive outcomes at 9 years after very preterm birth. Pediatrics 2012;129:e1138-47

6. Woodward LJ, Anderson PJ, Austin NC, et al. Neonatal MRI to predict neurodevelopmental outcomes in preterm infants. $N$ Engl J Med 2006;355:685-94

7. Nongena P, Ederies A, Azzopardi DV, et al. Confidence in the prediction of neurodevelopmental outcome by cranial ultrasound and MRI in preterm infants. Arch Dis Child Fetal Neonatal Ed 2010;95:F388-90

8. Ment LR, Hintz D, Hüppi PS. Imaging biomarkers of outcome in the developing preterm brain. Lancet Neurol 2009;8:1042-55

9. Arzoumanian Y, Mirmiran M, Barnes PD, et al. Diffusion tensor brain imaging findings at term-equivalent age may predict neurologic abnormalities in low birth weight preterm infants. AJNR Am J Neuroradiol 2003;24:1646-53

10. Hüppi PS, Dubois J. Diffusion tensor imaging of brain development. Semin Fetal Neonatal Med 2006;11:489-97

11. Liu Y, Aeby A, Baleriaux D, et al. White matter abnormalities are related to microstructural changes in preterm neonates at termequivalent age: a diffusion tensor imaging and probabilistic tractography study. AJNR Am J Neuroradiol 2012;33:839-45

12. Rose J, Butler EE, Lamont LE, et al. Neonatal brain structure on MRI and diffusion tensor imaging, sex, and neurodevelopment in verylow-birthweight preterm children. Dev Med Child Neurol 2009;51:526-35

13. Krishnan ML, Dyet LE, Boardman JP, et al. Relationship between 
white matter apparent diffusion coefficients in preterm infants at term-equivalent age and developmental outcome at 2 years. Pediatrics 2007;120:e604-09

14. van Kooij BJ, de Vries LS, Ball G, et al. Neonatal tract-based spatial statistics findings and outcome in preterm infants. AJNR Am J Neuroradiol 2012;33:188-94

15. Counsell SJ, Edwards AD, Chew ATM, et al. Specific relations between neurodevelopmental abilities and white matter microstructure in children born preterm. Brain 2008;131:3201-08

16. Bayley N. Bayley Scales of Infant and Toddler Development. 3rd ed. San Antonio, Texas: Psychological Corporation; 2005

17. Jiang H, van Zijl PC, Kim J, et al. DtiStudio: resource program for diffusion tensor computation and fiber bundle tracking. Comput Methods Programs Biomed 2006;81:106-16

18. Anjari M, Srinivasan L, Allsop JM, et al. Diffusion tensor imaging with tract-based spatial statistics reveals local white matter abnormalities in preterm infants. Neuroimage 2007;35:1021-27

19. Boardman JP, Craven C, Valappil S, et al. A common neonatal image phenotype predicts adverse neurodevelopmental outcome in children born preterm. Neuroimage 2010;52:409-14

20. Moore T, Johnson S, Haider S, et al. Relationship between test scores using the second and third editions of the Bayley Scales in extremely preterm children. J Pediatr 2012;160:553-58

21. Feldman HM, Lee ES, Yeatman JD, et al. Language and reading skills in school-aged children and adolescents born preterm are associated with white matter properties on diffusion tensor imaging. Neuropsychologia 2012;50:3348-62

22. Feldman HM, Lee ES, Loe IM, et al. White matter microstructure on diffusion tensor imaging is associated with conventional magnetic resonance imaging findings and cognitive function in adolescents born preterm. Dev Med Child Neurol 2012;54:809-14

23. Eikenes L, Lohaugen GC, Brubakk AM, et al. Young adults born preterm with very low birth weight demonstrate widespread white matter alterations on brain DTI. Neuroimage 2011;54:1774-85

24. Partridge SC, Mukherjee P, Henr RG, et al. Diffusion tensor imaging: serial quantitation of white matter tract maturity in premature newborns. Neuroimage 2004;22:1302-14

25. Ito M, Watanabe $H$, Kawai $Y$, et al. Usefulness of combined fractional anisotropy and apparent diffusion coefficient values for detection of involvement in multiple system atrophy. J Neurol Neurosurg Psychiatry 2007;78:722-28

26. Mori S, Zhang J. Principles of diffusion tensor imaging and its applications to basic neuroscience research. Neuron 2006;51:527-39

27. Budde MD, Kim JH, Liang HF, et al. Toward accurate diagnosis of white matter pathology using diffusion tensor imaging. Magn Reson Med 2007;57:688-95

28. Gupta RK, Hasan KM, Trivedi R, et al. Diffusion tensor imaging of the developing human cerebrum. J Neurosci Res 2005;81:172-78

29. Stadlbauer A, Ganslandt O, Buslei R, et al. Gliomas: histopathologic evaluation of changes in directionality and magnitude of water diffusion at diffusion-tensor MR imaging. Radiology 2006:240;803-10

30. Concha L, Livy DJ, Beaulieu C, et al. In vivo diffusion tensor imaging and histopathology of the fimbria-fornix in temporal lobe epilepsy. J Neurosci 2010;30:996-1002

31. Hüppi PS, Murphy B, Maier SE, et al. Microstructural brain development after perinatal cerebral white matter injury assessed by diffusion tensor magnetic resonance imaging. Pediatrics 2001;107: 455-60

32. Nagy A, Ashburner J, Andersson J, et al. Structural correlates of preterm birth in the adolescent brain. Pediatrics 2009;124:e964-72

33. Shim SY, Jeong HJ, Son DW, et al. Altered microstructure of white matter except the corpus callosum is independent of prematurity. Neonatology 2012;102:309-15

34. Pogribna U, Yu X, Burson K, et al. Perinatal clinical antecedents of white matter microstructural abnormalities on diffusion tensor imaging in extremely preterm infants. PLoS One. In press

35. Hack M, Taylor HG, Drotar D, et al. Poor predictive validity of the Bayley Scales of Infant Development for cognitive function of extremely low birth weight children at school age. Pediatrics 2005;116: $333-41$ 